\title{
PROPUESTA METODOLÓGICA PARA LA DEFINICIÓN DE ESTRATEGIAS DE MEJORAMIENTO EN LOGÍSTICA DE PYMES
}

\author{
(Methodological proposal for the definition of improvement strategies in logistics of SME)
}

\author{
Yeimy Liseth Becerra
}

Escuela de Ingeniería Industrial, Uptc. liseth.becerra@hotmail.com

(Recibido julio 19 de 2014 y aceptado septiembre 01 de 2014)

\begin{abstract}
Resumen:
Se presenta una propuesta metodológica para la definición de estrategias de mejoramiento en logística de pymes, como medio para el cumplimiento de un objetivo específico del proyecto Diseño metodológico sobre logística de almacenamiento, adquisición, apropiación de sistemas de información y comunicación para las pymes colombianas, subsector panificador, que actualmente ejecuta el grupo de investigación SEPRO, de la Universidad Nacional de Colombia y apoyado por Colciencias. El proyecto pertenece a la realización de la última etapa del proyecto base, y pretende dar cumplimiento al objetivo correspondiente, planteado en el proyecto de investigación que ha venido ejecutando el grupo de investigación SEPRO. Para ello, se hizo una revisión de la metodología empleada durante la ejecución del proyecto base, así como una elaboración de un estado de la cuestión de las técnicas empleadas en investigaciones similares para la evaluación y definición de estrategias de mejoramiento en logística de pymes. Se compararon las técnicas revisadas y se configuró una propuesta metodológica compuesta por las técnicas que representaron las mayores ventajas para el desarrollo de las investigaciones.
\end{abstract}

Palabras clave: cadena de suministro, logística, pymes, eficacia, integración, estrategias de mejoramiento.

\section{INTRODUCCIÓN}

El grupo de investigación Sociedad, Economía y Productividad SEPRO, línea de investigación Supply Chain Management-Logística, adscrito a la Universidad Nacional de Colombia, es un grupo multidisciplinar, con

\begin{abstract}
:
A methodological proposal for defining strategies of improvement in logistics of SMEs is presented as a means to fulfill a specific objective of the project Methodological design on storage logistics, acquisition, ownership of information systems and communication for Colombian SMEs, baker subsector, which currently runs the research group SEPRO, of Universidad Nacional of Colombia and supported by Colciencias.

The project corresponds to the completion of the last stage of the base project, and aims to implement the corresponding target, raised in the research project that has been developing the research group SEPRO. To do this, it was made a review of the methodology used during the execution of the basic project, as well as the state of the art of techniques used in similar research for the evaluation and definition of breeding strategies in SMEs logistics. Revised techniques were compared and a proposed methodology was configured, which consists of the techniques that represented the greatest advantages for the research development.
\end{abstract}

Keywords: supply chain, logistics, SMES, efficacy, integration.

profesionales con perfil doctoral, dedicado al estudio y aplicación de conceptos de la gestión de operación logística, que abarcan análisis transversales relacionados con tecnologías de información y comunicación, de automatización, finanzas, economía, medio ambiente, sociolo- 
gía y de políticas públicas, en el contexto de la cadena de abastecimiento-distribución.

El presente proyecto hace parte de los resultados esperados del proyecto Diseño metodológico sobre logística de almacenamiento, adquisición, apropiación de sistemas de información y comunicación para las pymes Colombianas, subsector panificador, actualmente en curso en el grupo SEPRO; por ello, la propuesta metodológica resultante es instrumento de referencia para llevar a cabo futuras investigaciones relacionadas, facilitando más el desarrollo de dichas actividades investigativas para el grupo de investigación, los empresarios y la comunidad académica en general.

\section{METODOLOGÍA}

Se desarrolla una investigación de tipo descriptivo, mediante las siguientes etapas:

2.1 Etapa 1: revisión, definición y documentación de las actividades llevadas a cabo durante la ejecución del proyecto base. En esta etapa se tomaron los documentos que han sido generados a través de la ejecución del proyecto Diseño metodológico sobre logística de almacenamiento, adquisición, apropiación de sistemas de información y comunicación para las pymes Colombianas, subsector panificador como productos, así como los diferentes instrumentos que han sido empleados y se configuró a nivel general la metodología que se ha venido utilizando, describiendo cada una de las actividades llevadas a cabo.

2.2 Etapa 2: realización de un estado de la cuestión de las técnicas empleadas para la evaluación y el diseño de propuestas de mejoramiento en logística. En esta etapa se hizo una revisión bibliográfica en diferentes bases de datos y libros relacionados, y se definieron los métodos que han sido utilizados en el desarrollo de investigaciones similares a la del proyecto, destacando las características principales de las mismas.

2.3 Etapa 3: comparación y análisis de los hallazgos de las revisiones realizadas. En esta etapa se compararon los hallazgos del estado de la cuestión y los de la metodología desarrollada a lo largo del proyecto Diseño metodológico sobre logística de almacenamiento, adquisición, apropiación de sistemas de información y comunicación para las pymes Colombianas, subsector panificador, con base en las ventajas y desventajas o limitaciones de los diferentes puntos contenidos en cada uno de los elementos generales de las metodologías estudiadas y se definió cuáles de estos formarían parte de la propuesta metodológica final.

2.4 Etapa 4: establecimiento y documentación de una propuesta metodológica compuesta por los procedimientos y elementos juzgados como más pertinentes, de acuerdo con el análisis efectuado. Como resultado de esta etapa se consolidó la metodología propuesta en un documento que servirá como guía para futuras investigaciones de este tipo, que sean ejecutadas por el grupo de investigación SEPRO, o cualquier otro grupo de investigación o empresarial al que pueda serle útil.

\section{GENERALIZACIÓN DEL PROCEDIMIENTO EMPLEADO EN EL DESARROLLO DEL PRO- YECTO BASE}

Para facilitar el estudio y comparación del procedimiento desarrollado durante la ejecución del proyecto base con metodologías halladas en la revisión bibliográfica que se expondrá posteriormente, se considera conveniente hacer una generalización de las actividades realizadas en el primero.

\subsection{Diagnóstico}

Para esta etapa, se realizan las siguientes actividades: i. planeación del estudio y definición del área de influencia, ii. definición de la estructura de la cadena de suministro, iii. determinación de la información requerida, iv. selección y acercamiento a las pymes que van a ser vinculadas al estudio, v. diseño y aplicación de instrumentos de recolección de información, teniendo en cuenta procesos de abastecimiento, almacenamiento, distribución y buenas prácticas de manufactura (además de la normatividad aplicable al sector), vi. recolección y análisis de información, vii. definición de indicadores logísticos de almacenamiento, producción, distribución y servicio, de acuerdo con la información recolectada.

\subsection{Planteamiento de oportunidades de mejora}

Se ejecuta a partir de los resultados obtenidos del estudio, los indicadores calculados y un modelamiento matemático, simulación de la cadena de suministros objeto de estudio y combinación de herramientas específicas para la optimización de actividades de los procesos logísticos 
llevados a cabo en la cadena de suministros estudiada, teniendo en cuenta su estructura y eslabones involucrados. Además se establecen buenas prácticas para los procesos de abastecimiento, almacenamiento producción y distribución de acuerdo con las normas técnicas y demás normatividad aplicable al sector y productos elaborados por las empresas objeto de estudio.

\subsection{Divulgación e implementación de las oportunidades de mejora}

Entrega y explicación de los resultados de la investigación y propuestas de mejoramiento definidas mediante las actividades anteriores a las empresas de la cadena de suministro objeto de estudio, brindando capacitación y acompañamiento para la implementación de las mismas.

\section{ESTADO DE LA CUESTIÓN DE LAS METO- DOLOGÍAS Y MODELOS EMPLEADOS PARA LA EVALUACIÓN Y DISEÑO DE PROPUESTAS DE MEJORAMIENTO EN LOGÍSTICA}

Se hizo una revisión bibliográfica en bases de datos especializadas, libros, instituciones de gobierno y organismos privados dedicados a estudios y actividades relacionadas con la logística.

Debe aclararse que se documentarán metodologías integrales para la definición de estrategias de mejoramiento en logística y cadena de suministros, metodologías parciales para el diagnóstico de la cadena, metodologías para mejoramiento de procesos específicos y herramientas de medición y mejoramiento de la cadena de suministros.

\subsection{Metodologías integrales}

4.1.1 Modelo SCOR. El modelo SCOR (Supply Chain Operations Reference Model, por su sigla en inglés), es un modelo de referencia de procesos, que provee un lenguaje de comunicación común a lo largo de los involucrados en la cadena de abastecimiento, y permite representar, analizar y configurar cadenas de suministro.

4.1.2 Modelo de gestión de la cadena de suministros. En el modelo de gestión de la cadena de suministros del Global Supply Chain Forum (Processes, Partnership, Performance) (Lambert, 2004), el GSCF identificó ocho procesos de negocio clave para manejar la cadena de su- ministros y establecer una forma de comunicación entre los diferentes eslabones.

4.1.3 Metodología CEBOR. La metodología CEBOR para el mejoramiento del sistema logístico desarrollada por la LALC (Latin America Logistics Center, 2003) permite administrar efectivamente sistemas logísticos en cualquier contexto y alcance y tiene las fases de conceptualización, evaluación, balanceo de entradas y salidas, optimización y reconfiguración del sistema.

4.1.4 Modelo tecnológico para el desarrollo de proyectos logísticos usando Lean Six Sigma. Este modelo desarrollado por Mantilla y Sánchez (2012) tiene el objetivo de orientar a las empresas a mejorar su desempeño logístico, para hacer más eficientes las operaciones logísticas a través de la reducción de la variabilidad y el desperdicio en la cadena de valor, ofreciendo un mejor servicio al cliente y a su vez reducir costos.

4.1.5 Lean Six Sigma Logistics. Lean Six Sigma Logistics (Goldsby \& Martichenko, 2005) se puede definir como la eliminación de los desperdicios a través de esfuerzos disciplinados para entender y reducir la variación, mientras se incrementa la velocidad y el flujo en la cadena de suministro.

4.1.6 Vinculación de la estrategia y procesos de la cadena de suministros para mejoramiento del desempeño. El modelo propuesto (Alomar \& Pasek, 2014) ayudará a las empresas, particularmente pymes, a evaluar su desempeño privilegiando procesos de la cadena de suministros y seleccionando una estrategia adecuada bajo varios escenarios de mercado.

4.1.7 Metodología de gestión logística para el mejoramiento de pequeñas empresas. Se presenta una metodología (González, Flores, Cervera \& Arroyo, 2013) de apoyo para mejorar las operaciones logísticas de los pequeños negocios, teniendo en cuenta este tipo de empresas generalmente no cuentan con los recursos económicos para pagar una consultoría en logística.

4.1.8 Metodología para el análisis de las cadenas logísticas en el transporte multimodal en Sudamérica. Los objetivos de la metodología para el análisis de las cadenas logísticas en el transporte multimodal en Sudamérica (Moiraghi, 2006) son identificar oportunidades de diversificación y especialización de servicio logísticos que 
promuevan la eficiencia o agregación de valor para los segmentos target identificados y definir los incentivos adecuados para ser generados por parte de los sectores públicos o privados para provisión de dicha oferta.

\subsection{Metodologías parciales para el diagnóstico de la ca- dena de suministros}

4.2.1 Una metodología de diagnóstico para la distribución urbana de mercancías. En el documento titulado Una metodología de diagnóstico para la distribución urbana de mercancías: caso de estudio en ciudad de Belo Horizonte (Brasil) (De Oliveira \& Dias, 2012) se presenta una metodología para identificar aspectos relacionados a la distribución urbana de mercancías, para lo cual se realizaron entrevistas con operadores logísticos, se investigaron sitios de cargue y descargue y cuentas de tráfico en el área de estudio.

4.2.2 Herramienta de auditoría para políticas de eficiencia logística. Se presenta un acercamiento a formulación de políticas en el campo de logística (Bozicnik, Letnik \& Stiglic, 2012), desarrollado dentro de un proyecto denominado CASTLE. Este da un ejemplo de una herramienta de política para la auditoría en logística y procesos de gestión de materiales y procedimientos en pymes, intentando proveer entradas cruciales para la formulación de políticas.

4.2.3 Tamaño de la empresa y el rendimiento sostenible en las cadenas de suministro de alimentos. En el documento denominado Tamaño de la empresa y el rendimiento sostenible en las cadenas de suministro de alimentos: Perspectivas de las PYMES griegas (Bourlakis, Maglaras, Aktas \& Gallear, 2013) se analiza las diferencias en el desarrollo sostenible dentro de la cadena de suministros de alimentos griega y provee numerosas comparaciones estadísticas de sus miembros clave (cultivadores, procesadores, mayoristas y minoristas) con respecto al tamaño de la firma.

4.2.4 Metodología para la evaluación del rendimiento de la cadena logística. Se presenta una metodología para la construcción de un sistema de evaluación del rendimiento de la cadena logística integral, denominada MESIADLog (Campos, Taboada \& Chalmeta, 2004), la cual fue aplicada en empresas brasileñas y españolas para validarla y permite a las empresas elaborar su propio sistema de evaluación logística integrado.
4.2.5 Metodología para la gestión del desarrollo de servicios logísticos de valor agregado IIRSA. La metodología de la Iniciativa para la Integración de la Infraestructura Regional Sudamericana (IIRSA) (Advanced Logistics group, 2006) se aplica al análisis macro de redes y no a la logística micro enfocada en una industria, de ahí la importancia que se da a los componentes de carácter estratégico, funcionales y de planificación.

4.3 Metodologías para mejoramiento de procesos especificos

4.3.1 Mejoramiento del cargue en el despacho de un centro de distribución utilizando superficies de respuesta. Este estudio para el mejoramiento del cargue en el despacho de un centro de distribución utilizando superficies de respuesta (Gómez \& Correa, 2012) tiene por objetivo utilizar la técnica de optimización de diseño de experimentos denominada metodología de la superficie de respuesta para reducir el tiempo de cargue en la operación de despacho, lo cual contribuye con la eficiencia del centro de distribución (CEDI).

4.3.2 Identificación de oportunidades de mejora en la gestión del transporte del carbón en Colombia con Six Sigma. Se presenta una aplicación de six sigma como técnica que facilita la identificación de oportunidades de mejora en la gestión del transporte del carbón (Arango, Gómez \& Álvarez, 2011) con el objetivo de obtener un desarrollo eficiente y buscando la satisfacción de las necesidades de los clientes.

4.3.3 Diseño basado en logística como una aproximación a desarrollo de despachos y negocios. Presenta una metodología y herramientas que consideran los requerimientos y expectativas de todas las partes interesadas de la cadena de transporte, permitiendo el diseño de una mayor eficiencia en la operación de despacho como parte de cadenas de transporte intermodal dinámicas (Boulougouris, Papanikolaou, Ostvik, Brett \& Konovessis, 2012). La metodología se desarrolla dentro de un proyecto de investigación colaborativa denominado LOGBASED, cuyo objetivo fue desarrollar sistemas de transporte y despacho que contribuyeran a que los transportistas vía marítima fueran competitivos respecto a los transportistas terrestres.

4.3.4 La cadena de suministros alimentaria del Reino Unido: mejorando la eficiencia en la red logística. En el documento de Fernie y McKinnon (2003) se afirma que 
a pesar de que la cadena de suministros de la industria alimentaria del Reino Unido es una de las más eficientes del mundo, se considera que pueden realizarse mejoras al respecto, especialmente en la reducción de costos.

4.4 Herramientas para la medición de desempeño y mejoramiento de la cadena de suministros

4.4.1 Lean Six Sigma en pequeñas y medianas empresas: un enfoque metodológico. Se propone una metodología para la implementación de un enfoque integrado Lean Six Sigma (LSS) (Felizzola \& Amaya, 2014), el cual se adapta a las necesidades y características de las pymes.

4.4.2 Conceptos estructurales para la cooperación horizontal para aumentar la eficiencia en logística. Dado que la cooperación logística horizontal entre los transportistas es un nuevo y poderoso método para optimizar las estructuras de costos y está ganando impulso en empresas individuales con el fin de sobrevivir en mercados competitivos, esta publicación se ocupa del diseño conceptual y los aspectos organizacionales de la cooperación horizontal.

4.4.3 Calidad del servicio logístico: modelo conceptual y evidencia empírica. Se explora la definición del concepto de calidad en el servicio logístico y sus dimensiones asociadas para construir un modelo conceptual y evaluarlo empíricamente (Vinh, 2013).

4.4.4 Diseño de un modelo de gestión logística para pymes de industria textil. Se presenta una investigación descriptiva que emplea información secundaria como base para el diseño de un modelo de gestión logística para pymes que permite integrar recursos, habilidades y sistemas requeridos para mejorar su desempeño como parte de una cadena de abastecimiento (Cano, Orue, Martínez \& Mavett, 2013).

4.4.5 Características de un modelo de negocio basado en logística. Explora las características de un modelo de negocio basado en logística (Sandberg, Kihlén \& Mats, 2011), fundamentado en un caso de estudio múltiple sobre tres compañías minoristas nórdicas, las cuales tienen crecimiento y rentabilidad superior al promedio de su industria, crecimiento coherente, poseen un enfoque estratégico en logística para la gestión.
4.4.6 Medición del desempeño de la gestión de la cadena de suministros: una aproximación a Balanced Score Card. Se desarrolla un cuadro de mando integral (Balanced Score Card, BSC) para la gestión de la cadena de suministros (SCM) que mide y evalúa las operaciones del día a día del negocio desde cuatro perspectivas: finanzas, cliente, procesos de negocio internos y aprendizaje y crecimiento (Bhagwat \& Sharma, 2007).

\section{COMPARACIÓN Y ANÁLISIS DE LOS HA- LLAZGOS DE LAS REVISIONES REALIZADAS}

En esta etapa se compararon los hallazgos del estado de la cuestión, con la metodología desarrollada a lo largo del proyecto Diseño metodológico sobre logística de almacenamiento, adquisición, apropiación de sistemas de información y comunicación para las pymes colombianas, subsector panificador, con base en las fortalezas y debilidades o limitaciones de cada uno de los procedimientos; para esto se tuvieron en cuenta los elementos fundamentales propios de cada método o modelo estudiado (de fases metodológicas, de procesos específicos objeto de estudio y recursos para la aplicación de la metodología), se destacaron los que podrían ser parte de la metodología final por su claridad y facilidad de aplicación, aporte a la eficacia de las investigaciones referentes a la definición de mejoras logísticas de pymes, así como su importancia para asegurar que se lleve a cabo un correcto diagnóstico, planeación y ejecución de oportunidades de mejora en las cadenas de suministro objeto de estudio y las empresas que conforman cada eslabón de dichas cadenas. Teniendo en cuenta lo anterior, fueron descartados aquellos elementos que no contribuían al objetivo o no presentaban las características requeridas, lo cual permitió seleccionar los elementos que formarían parte de la metodología propuesta.

\section{DEFINICIÓN DE LA PROPUESTA METODO- LÓGICA}

A continuación se describe la propuesta metodológica para la definición de estrategias de mejoramiento en logística de pymes, a partir de los elementos que resultaron más adecuados de acuerdo con el análisis efectuado; se enmarcó la propuesta metodológica en las fases de la herramienta DMAIC (definir, medir, analizar, mejorar y controlar) y se definieron las actividades específicas propias de cada fase. 


\subsection{Fase de definición}

Esta fase está conformada por las etapas siguientes:

6.1.1 Conceptualización del sistema. Se busca definir tanto el alcance funcional como el territorial de la cadena que se va a analizar. Se definen fronteras de magnitud de la cadena, los agentes o entidades que la conforman, la capacidad de las actividades, el contexto de operación y los flujos y relaciones entre los agentes que componen la cadena. Durante esta etapa se logra entender y relacionar los elementos estructurales que caracterizan cada uno de los eslabones y permiten una apreciación general de la capacidad de la red logística ante las exigencias de los consumidores.

6.1.2 Identificación y delimitación del problema. Se identifica, describe y establece el alcance del problema, el cual debe orientarse a mejorar la eficiencia de la cadena, uno o varios de sus eslabones en uno o varios aspectos o procesos específicos. En caso de mejoramiento global de la cadena, se deben establecer las prioridades sobre las áreas, procesos, productos o servicios críticos, se debe trabajar para la consecución de los objetivos planteados, según el tiempo, duración, costo, complejidad e impacto en el cliente.

6.1.3 Planeación del trabajo de campo. Se deben definir los métodos y recursos que se van a emplear para la recolección de información primaria y secundaria de la cadena objeto de estudio y la información requerida para realizar un diagnóstico preciso de la situación logística de la cadena de suministros, teniendo en cuenta generalidades de las empresas (planeación estratégica, grado de compromiso de la dirección con los recursos y el impulso requerido para la implementación de posibles oportunidades de mejora detectadas, grado de estandarización de los procesos de la empresa), los principales procesos logísticos (abastecimiento, almacenamiento, distribución) y el cumplimiento de la normatividad aplicable al sector específico de las empresas que conforman la cadena. Además, se debe definir la cantidad de empresas de cada eslabón que formarán parte del estudio y seleccionarlas dentro del grupo específico de empresas que componen la cadena.

6.1.4 Acercamiento a las pymes objeto de estudio. Una vez identificadas las empresas para auditar, se debe hacer un acercamiento a estas, informando sobre los objetivos y alcance del estudio por realizar y pidiendo autorización pertinente para la ejecución del mismo.

6.2 Fase de medición. Se aplican los instrumentos diseñados, los cuales generalmente son entrevistas o encuestas aplicadas a los administradores, gerentes o propietarios del grupo o muestra de empresas que forman parte de estudio teniendo en cuenta los aspectos por evaluar definidos; deberían incluirse la medición de las siguientes características (las que apliquen a las empresas estudiadas):

-En soporte estratégico y de operaciones: presupuesto, planificación estratégica, desarrollo de recursos humanos, y soporte de adquisiciones, planificación de ventas y operaciones, entrenamiento y desarrollo, comprensión y aplicación de un uso efectivo de la tecnología y gestión de datos, eliminación de los desperdicios y ejecución de eficiencias operativas.

Se debe verificar si se están ejecutando las siguientes actividades principales: estimación de la demanda, definición de política de precios, definición de niveles de servicio óptimos, definición de cantidades óptimas de reposición de inventarios, selección y gerencia de proveedores, evaluación de fuentes de abastecimiento, selección y gerencia de distribuidores y gestión de flota de transporte.

-En cuanto a planeación estratégica: se requiere verificar si se tiene definida una misión, visión y objetivos de la empresa a corto, medio y largo plazo, con el fin de determinar una dirección de la organización que sea base para garantizar la efectividad de cualquier iniciativa de mejoramiento que se emprenda.

Se tuvieron en cuenta los elementos del nivel tres de SCOR (se pueden complementar con los elementos de planeación de la metodología CEBOR). En primer lugar se debe identificar la configuración de trabajo bajo la cual opera la empresa (para stock, para pedido o diseño de pedido, o para ambos) y a continuación seleccionar los elementos por verificar de cada uno de los procesos clave, que, en síntesis, son los siguientes:

-En el proceso de compras: la forma, medios empleados y costos de identificar proveedores, seleccionar proveedor final y negociar, programar envíos de productos, recepción de producto, verificación de producto. 
-En el proceso de producción: forma, medios empleados y costos de diseñar un producto, si se requiere, programar las actividades de producción, liberar productos comprados o en proceso, producir y probar, embalar y almacenar el producto terminado y liberarlo para su distribución. Este proceso solo aplica a pymes manufactureras; para empresas de servicios se podrían adaptar los elementos a fin de hacerlos coincidir con los necesarios para la prestación de un servicio: forma, medios empleados y costos de diseñar el servicio si se requiere, programar las actividades de prestación del servicio, prestar el servicio.

-En el proceso de almacenamiento: estratificación, ubicación de inventarios, pronósticos, procesamiento de pedidos.

-En el proceso de entrega: forma, medios empleados y costos de negociar y recibir contratos, ingresar un pedido, "rutear" despachos si se hace, recibir productos de compras o manufactura, recolectar productos, el embalaje de los mismos, cargar producto y generar documentos de envío, enviar o entregar el producto al cliente y realizar la respectiva facturación.

-En el proceso de devolución: forma, medios empleados y costos de identificar la condición de los productos en exceso o defectuosos, disponer el producto en exceso o defectuoso, requerir devolución programar envío de producto en exceso o defectuoso.

\subsection{Fase de análisis}

En esta etapa se observa la composición de la cadena, su grado de madurez y desempeño logístico, se organiza y depura la información y los datos obtenidos en campo.

6.3.1 Diagnóstico de procesos. Se analiza la información recolectada, a través de herramientas estadísticas de análisis, modelos matemáticos, simulación y demás métodos de los que se pueda disponer y apliquen al tipo y nivel de profundidad del estudio que se está realizando. Este análisis de aspectos cualitativos permitirá una visualización del grado de eficiencia de los diferentes procesos logísticos en las empresas estudiadas.

Para efectuar este diagnóstico se debería desarrollar en primer lugar un diagnóstico estratégico de las empresas, el cual establece si las empresas y la cadena de suminis- tros tienen un enfoque claro y una dirección estratégica de sus actividades, que es fundamental para garantizar el éxito y la sostenibilidad; para esto es conveniente detallar separadamente las actividades de planeación y ejecución de las empresas objeto de estudio, teniendo en cuenta los elementos de proceso del nivel 3 del modelo SCOR, que se pueden complementar con los elementos de planeación de la metodología CEBOR.

Por otra parte, se debe analizar el nivel de alineación entre suministro y demanda, las tecnologías de información empleadas por las empresas, los flujos físicos y objetivos de las mismas, el grado de enfoque que tienen en el cliente, los datos y los proveedores.

6.3.2 Definición de indicadores de desempeño logísticos. Teniendo como fuente la información recolectada en la fase de medición, se definen indicadores de desempeño logístico en cada uno de los procesos estudiados, los cuales son un elemento imprescindible para el control de los procesos, que aseguren la sostenibilidad de las empresas en el tiempo.

\subsection{Fase de mejoramiento}

6.4.1 Planteamiento de oportunidades de mejoramiento. De acuerdo con el análisis realizado, se deben plantear acciones de mejora que estén encaminadas a la eficiencia logística y calidad del servicio en toda la cadena, considerando las limitaciones de recursos en general que presentan las pymes, que debe comenzar con la definición de un plan estratégico organizacional de las empresas, si estas no cuentan con uno y un entrenamiento efectivo del personal en aspectos de logística y en particular los planes de acción que sean definidos; se deben tener en cuenta cada uno de los elementos y aspectos que forman parte de las anteriores fases para asegurar que las estrategias de mejora se definan correctamente. Una herramienta de utilidad para plantear las oportunidades de mejora es la simulación y el modelamiento matemático.

6.4.2 Divulgación e implementación de oportunidades de mejora. Se deben divulgar los resultados de la investigación realizada a las empresas y personas pertinentes, exponiendo la situación de cada empresa en cuanto a sus procesos logísticos y de gestión de la cadena de suministros de acuerdo con el diagnóstico realizado y los indicadores definidos, así como las alternativas de mejoramiento propuestas en cada uno de los aspectos, brindando apoyo y capacitación si es necesaria para su 
implementación. Debe ponerse de manifiesto que el compromiso por parte de la dirección en la implementación de las estrategias de mejoramiento planteadas es de gran importancia para asegurar el éxito de dichas estrategias, que redundarán en una mayor eficiencia, competitividad y productividad de las empresas y la cadena de suministro estudiada.

\subsection{Fase de control}

Se requiere definir y aplicar mecanismos de control y monitoreo de las mejoras realizadas, analizar periódicamente si se cumplen los plazos establecidos para la ejecución de las oportunidades de mejora planeadas, identificar las tareas realmente cumplidas, el responsable de su ejecución y la fecha en que se realizaron; es necesario que se lleve a cabo un control cuantitativo del desempeño de cada proceso a través del monitoreo constante de los indicadores logísticos definidos y de las variables de cada proceso específicas que requieran control para garantizar la calidad en el servicio logístico y del producto o servicio entregado al cliente. Además se debe hacer seguimiento a los impactos generados por las estrategias propuestas, en la consecución de los objetivos planteados y la respuesta generada en el cliente, ajustando las actividades en caso de no cumplimiento o detección de nuevas oportunidades de mejora y creando un nuevo plan de acción para garantizar que se ejecuten las medidas propuestas.

\section{CONCLUSIONES}

Las actividades y productos del proyecto base fueron documentados, indicando elementos de evaluación y mejoramiento de la cadena de suministros que se conservaron en gran parte en la propuesta metodológica definitiva, debido a que presentaron similitud y coherencia con la mayoría de las metodologías que fueron revisadas en distintas fuentes bibliográficas.

Se llevó a cabo una revisión bibliográfica de técnicas empleadas para la evaluación y diseño de propuestas de mejoramiento en logística y se observó que los diferentes autores desarrollan metodologías con herramientas, procesos y actividades acordes con el enfoque de un estudio particular, por lo cual la generalización de los elementos se hizo con base en los aspectos fundamentales de la gestión de la cadena de suministros.
Se analizaron las ventajas y desventajas de los diferentes elementos de las metodologías revisadas, hallando que algunos de ellos no son convenientes para las pymes, debido a que implican la inversión de recursos con los cuales difícilmente cuenta este tipo de empresas y por ello fueron descartados de la metodología propuesta.

Se definió la propuesta metodológica con base en la metodología DMAIC de Seis Sigma, lo cual facilitó la inclusión de los diferentes elementos para la gestión de la cadena de suministros, que incluyeron guías de indicadores, mejores prácticas y herramientas de diagnóstico $\mathrm{y}$ toma de decisiones que pueden soportar iniciativas de mejoramiento logístico en cualquier sector productivo.

Las pymes presentan varias limitaciones para la aplicación de estrategias de mejoramiento en el área logística, por lo cual se hace necesario un acompañamiento de diferentes entidades académicas y gubernamentales, así como pactos empresariales para ayudar a que estas empresas hagan una mejor gestión de la cadena de suministros y logística y por ende aumenten su productividad y competitividad; lo anterior se puede facilitar a través del empleo de la metodología propuesta en investigaciones al respecto.

Como elementos fundamentales para la definición de estrategias de mejoramiento en logística de pymes se identificó el empleo de indicadores de gestión logística, el uso de mejores prácticas sugeridas por diferentes autores, y la aplicación de adecuadas herramientas de diagnóstico y toma de decisiones.

\section{REFERENCIAS}

Advanced Logistics Group. (2006). Metodología de Análisis del Potencial de Integración Productiva y desarrollo de servicios logísticos de valor agregado en corredores IIRSA. Libro Final. Tomo 3. Apéndice I. Argentina.

Alomar, M., \& Pasek, Zbigniew J. (2014). Linking supply chain strategy and processes to performance improvement. Procedia CIRP 17,628-634.

Arango, M.D., Gómez, R.A., \& Álvarez, K.C. (2011, dic.). Identificación de oportunidades de mejora en la gestión del transporte del carbón en Co- 
lombia con Six Sigma. Boletín de Ciencias de la Tierra, (30), 23-38.

Arcila, A., Buitrago, C. \& Muñoz. L (2005). Estudio de la cadena de abastecimiento del sector farmacéutico veterinario con base en el modelo SCOR. Trabajo de grado. Universidad de Los Andes.

Arias, H. (2013). Manipulación inocua de insumos para el subsector panificador de Palmira Valle, en las operaciones de transporte, recepción y almacenamiento. Trabajo de grado. Universidad Nacional de Colombia.

Ballesteros, C. (2013). Construcción de una línea base de información logística de las pymes subsector panificador. Contexto municipio de Palmira. Trabajo de grado. Universidad Nacional de Colombia, sede Palmira.

Ballou, R. H. (2004). Logística, Administración de la cadena de suministro. (5 ed.). México: Pearson Educación.

Ballou, R. H., Gilbert, S. M. \& Mukherjee, A. (2000). New managerial challenges from supply chain. Industrial Marketing Management, 7-18.

Bhagwat, R., \& Kumar, M. S. (2007). Performance measurement of supply chain management: a balanced scorecard approach. Computers \& Industrial Engineering 53, 43-62.

Boulougouris, E., Papanikolaou, A., Ostvik, I., Brett, P.O., \& Konovessis, D. (2012). Diseño basado en logística como una aproximación a desarrollo de despachos y negocios. Procedia - Social and Behavioral Sciences, 48, 2241-2250.

Bourlakis,M., Maglaras, G., Aktas, E., Gallear, D. \& Fotopoulos, Ch. (2013). Firm size and sustainable performance in food supply chains: Insights from Greek SMEs. Int. J. Production Economics 152, 112-130.

Bozicnik, S., Letnik T. \& Stiglic, M. (2012). Audit tool for efficient logistics policy. Procedia, Social and Behavioral Sciences 48, 2967-2977.
Calderón, J. \& Lario, E. (2005). Análisis del modelo SCOR para la gestión de la cadena de suministro. En $9^{\circ}$ Congreso de Ingeniería de Organización, Gijón.

Campos, J., Taboada, C. \& Chalmeta, R. (2004). Metodología para la evaluación del rendimiento de la cadena logística. Inf. tecnol., 15(4).

Cano, P., Orue, F., Martínez, J. \& Mavett, Y. (2013). Design of a logistics management model for SMEs of textile manufacture. Global Conference on Business and Finance Proceedings. 8(1).

Cárdenas, I. (2013).Coordinación de agentes en cadenas de suministro descentralizadas, caso de estudio sector panificador, Palmira, Valle. Trabajo de grado. Universidad Nacional de Colombia.

Creswell, J. (2008). Educational research: planning, conducting, and evaluating quantitative and qualitative research. Upper Saddle River, NJ: Prentice Hall.

Chopra, S. \& Meindl, P. (2008). Admnistración de la cadena de suministro; estrategia, planeación y operación. (3 ed.). México: Pearson Educación.

Felizzola, H., \& Amaya, C.L. (2014). Lean Six Sigma en pequeñas y medianas empresas: un enfoque metodológico. Ingeniare. Revista chilena de ingeniería, 22(2), 263-277.

Fernie, J. \& McKinnon, A. (2003). The grocery supply chain in the UK: improving efficiency in the logistics network. The International Review of Retail, Distribution and Consumer Research, 13(2), 161-174.

Goldsby, T. \& Martichenko, R. (2005). Lean Six Sigma Logistics. Boca Ratón, Florida: J. Ross Publishing.

González, C.A., Martínez, J.L., Malcón Cevera, C., y Cavazos Arroyo, J. (2013). Metodología de gestión logística para el mejoramiento de pequeñas empresas. Revista Internacional Administración \& Finanzas, 6(5), 121-129. 
Gutiérrez, I. (2006). Estudio de la cadena de suministros del sector farmacéutico de plantas medicinales en Colombia con base en el modelo SCOR. Trabajo de grado. Universidad de los Andes.

Gómez Montoya, R.A. \& Correa Espinal, A.A. (2012). Mejoramiento del cargue en el despacho de un centro de distribución utilizando superficies de respuesta. Revista Lasallista de investigación, 9 (1), 70-85.

Houlihan, J. B. (1987). International supply chain management. International Journal of Physical Distribution \& Logistic Management, 51-66.

Lambert M, D. (2004). Supply chain management (processes, partnership, performance). United States of America: The Hartley Press, Inc.

Latin America Logistics Center (2003). Estrategia corporativa de logística, operaciones y redes de abastecimiento. Alta Dirección en Logística, Operaciones y Gerencia de Redes de Abastecimiento ADLOG.

Leitner, R., Meizer, F., Prochazka, M., \& Sihn, W. (2011). Structural concepts for horizontal cooperation to increase efficiency in logistics. CIRP Journal of Manufacturing Science and Technology 4, 332-337.doi: 10.1016/j.cirpj.2011.01.009

Mantilla, O.L., \& Sánchez, J.M. (2012, jul-sep.). Modelo tecnológico para el desarrollo de proyectos logísticos usando Lean Six Sigma. Estudios Gerenciales, 28(124), 23-43.
Moiraghi, L.E. (2006). Metodología para el análisis de las cadenas logísticas en el transporte multimodal en Sudamérica. Corrientes, Argentina: Universidad Nacional del Nordeste, Comunicaciones Científicas y Tecnológicas.

Simchi-Levi, D., Kaminsky, S. \& Simchi-Levi, E. (2000). Designing and managing the supply chain: concepts, strategies and cases. New York: McGraw-Hill.

Stewart, G. (1997). Supply-chain operations reference model (SCOR): the first cross-industry framework for integrated supply-chain management. Logistics Information Management, 10(2), 62-67.

Sánchez, J. (2010). Logística de una pyme. Revista de Logística, 9.

Sandberg, E., Kihlén,T. \& Abrahamsson,M. (2011). Characteristics of a Logistics-Based Business Model. Journal of Marketing Channels, 18(2), 123-145.

Supply-Chain Operations (2008). Reference model. SCOR. Overview Version 9.0 Supply-Chain Council.

Vinh, T. (2013). Logistics service quality: conceptual model and empirical evidence. International Journal of Logistics Research and Applications: A Leading Journal of Supply Chain Management, 16(2), 114-131. 